BMJ Open Sport \& Exercise Medicine

\section{Do the combined blood pressure effects of exercise and antihypertensive medications add up to the sum of their parts? A systematic meta-review}

To cite: Pescatello LS, Wu Y, Gao S, et al. Do the combined blood pressure effects of exercise and antihypertensive medications add up to the sum of their parts? A systematic meta-review. BMJ Open Sport \& Exercise Medicine 2021;7:e000895. doi:10.1136/ bmjsem-2020-000895

- Additional material is published online only. To view, please visit the journal online (http://dx.doi.org/10.1136/ bmjsem-2020-000895).

Accepted 30 December 2020

Check for updates

(c) Author(s) (or their employer(s)) 2021. Re-use permitted under CC BY-NC. No commercial re-use. See rights and permissions. Published by BMJ.

${ }^{1}$ Department of Kinesiology, University of Connecticut, Storrs, Connecticut, USA

${ }^{2}$ Department of Statistics, University of Connecticut, Storrs, Connecticut, USA

${ }^{3}$ Wesleyan University, Middletown, Connecticut, USA ${ }^{4}$ ICF Next, Fairfax, Virginia, USA

Correspondence to Dr Linda S Pescatello; Linda.Pescatello@uconn.edu

\section{ABSTRACT}

Objective To compare the blood pressure (BP) effects of exercise alone (EXalone), medication alone (MEDSalone) and combined (EX+MEDScombined) among adults with hypertension.

Data sources PubMed, Scopus, Cumulative Index to Nursing and Allied Health Literature, SPORTDiscus and the Cochrane Library.

Eligibility criteria Randomised controlled trails (RCTs) or meta-analyses (MAs) of controlled trials that: (1) involved healthy adults>18year with hypertension; (2) investigated exercise and $\mathrm{BP}$; (3) reported preintervention and postintervention BP and (4) were published in English. RCTs had an EX+MEDScombinedarm; and an EXalone arm and/or an MEDSalone arm; and MAs performed moderator analyses. Design A systematic network MA and meta-review with the evidence graded using the Physical Activity Guidelines for Americans Advisory Committee system.

Outcome The BP response for EXalone, MEDSalone and EX+MEDScombined and compared with each other. Results Twelve RCTs qualified with 342 subjects $(60 \%$ women) who were mostly physically inactive, middleaged to older adults. There were 13 qualifying MAs with 28468 participants ( $50 \%$ women) who were mostly Caucasian or Asian. Most RCTs were aerobic (83.3\%), while the MAs involved traditional (46\%) and alternative (54\%) exercise types. Strong evidence demonstrates EXalone, MEDSalone and EX+MEDScombined reduce

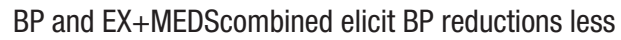
than the sum of their parts. Strong evidence indicates EX+MEDScombined potentiate the BP effects of MEDSalone. Although the evidence is stronger for alternative than traditional types of exercise, EXaloneelicits greater BP reductions than MEDSalone.

Conclusions The combined BP effects of exercise and medications are not additive or synergistic, but when combined they bolster the antihypertensive effects of MEDSalone.

PROSPERO registration number The protocol is registered at PROSPERO CRD42020181754.

\section{INTRODUCTION}

Hypertension is the most predominant risk factor for cardiovascular disease (CVD)

\section{What is already known?}

Exercise and medications alone and combined are all effective in reducing blood pressure.

\section{What are the new findings?}

- The combined blood pressure effects of exercise and medication do not equal the sum of their parts so that they are not additive.

- When exercise is combined with medication, they bolster the blood pressure effects of medication alone.

- Exercise alone elicits greater blood reductions than medication alone, evidence that is stronger for alternative than traditional forms of exercise.

affecting nearly one in two adults in the $\mathrm{USA}^{1}$; while CVD is the leading cause of death and disability in the USA and world accounting for one in three deaths. ${ }^{2}$ Zanchetti et $a \hat{l}$ performed a meta-analysis (MA) of randomised controlled trials (RCTs) and found antihypertensive medication use reduced all major types of cardiovascular events and death. Indeed, pharmacological treatment generated reductions in systolic blood pressure (SBP) of $9-10 \mathrm{~mm} \mathrm{Hg}$ and diastolic BP (DBP) of 4-5 mm Hg decreased the relative risk for all-cause mortality by $11 \%$, cardiovascular morality by $16 \%$, coronary heart disease by $18 \%$ and stroke by $36 \%$. Exercise is also recommended as antihypertensive lifestyle therapy because it lowers BP 5-8 $\mathrm{mm} \mathrm{Hg}$ among adults with hypertension, ${ }^{4}$ prevents the development of hypertension ${ }^{4-7}$ and reduces cardiovascular morbidity and mortality. ${ }^{8}$ Despite the well-established BP benefits of antihypertensive medications and exercise to prevent, treat and control hypertension, little is known about their 
comparative BP effects alone and combined in the treatment of hypertension.

Two recent network MAs (NMA) addressed this important knowledge gap with slightly different findings. Naci et al were the first to compare the BP lowering effects of exercise vs medications on SBP among healthy adults with normal BP, prehypertension and hypertension. ${ }^{9}$ They found aerobic and dynamic resistance exercise performed alone or combined reduced SBP by $\sim 9 \mathrm{~mm}$ $\mathrm{Hg}$ among adults with hypertension, with no detectable difference in the magnitude of the SBP reductions to those resulting from antihypertensive medications. ${ }^{9}$ Noone et al were the second to compare the BP-lowering effects of exercise and medications among adults with hypertension on BP in RCTs with a sample size of $\geq 20$ participants. ${ }^{10}$ They concluded there was insufficient evidence to suggest antihypertensive medications were better than exercise in lowering BP. Despite the efforts of these two investigative teams to directly compare the effectiveness of exercise and medication as antihypertensive therapeutic options, the authors acknowledged the exercise arms contained participants treated with medication so that the BP effects reported were not necessarily due to exercise per se, and RCTs that directly compared exercise to medications were not included.

We now perform an NMA of the primary literature and a meta-review of the secondary literature to answer the question: Do the BP effects of exercise and medication combined add up to the BP effects of the sum of their parts among adults with hypertension? The first search involved primary level RCTs that isolated and then directly compared exercise and medication combined (EX+MEDScombined) to exercise (EXalone) and/ or medication (MEDSalone) alone among adults with hypertension; and the second involved MAs of RCTs and controlled trials that examined the relationship between exercise and $\mathrm{BP}$ among adults with hypertension and reported moderators of the $\mathrm{BP}$ response to exercise.

\section{METHODS}

This systematic review is consistent with the Preferred Reporting Items for Systematic Reviews and MetaAnalyses statement. ${ }^{11} 12$

\section{Primary literature search strategy and selection process}

After consultation with a medical librarian (JL), we ran comprehensive Boolean searches in PubMed, Scopus, Cumulative Index to Nursing and Allied Health Literature (CINAHL), SPORTDiscus and the Cochrane Library from 1940 to 21 July 2016 and updated this search in PubMed and Scopus from 21 July 2016 to 26 February 2020. See supplemental digital content 1 (online supplemental SDC 1) for the original and updated search strategy and figure 1 for the selection process. Included studies were RCTs that: (1) involved healthy adults >18year with hypertension; (2) had an exercise, non-diet group put on antihypertensive medications (EX+MEDScombined) as part of the intervention arm (3) had an exercise, non-diet group not on antihypertensive medications (EXalone) and/or an non-exercise, non-diet

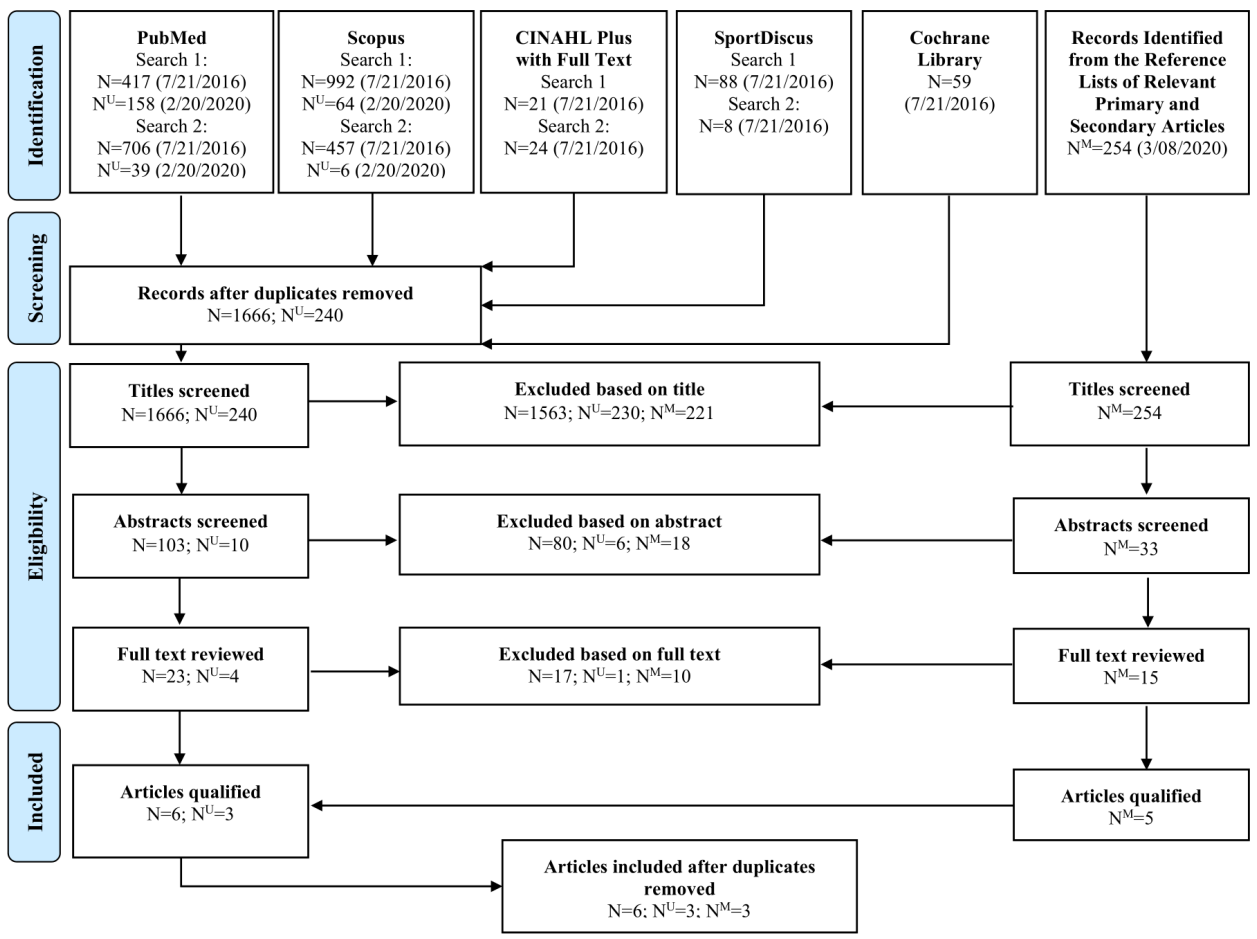

Figure 1 A summary of the primary literature systematic original and updated search selection process. $\mathrm{N}=$ The number of potentially qualifying articles from the original search. $\mathrm{N}^{\mathrm{U}}=$ The number of potentially qualifying articles from the updated search. $\mathrm{N}^{\mathrm{M}}=$ The number of potentially qualifying articles from the manual search. CINAHL, Cumulative Index to Nursing and Allied Health Literature. 


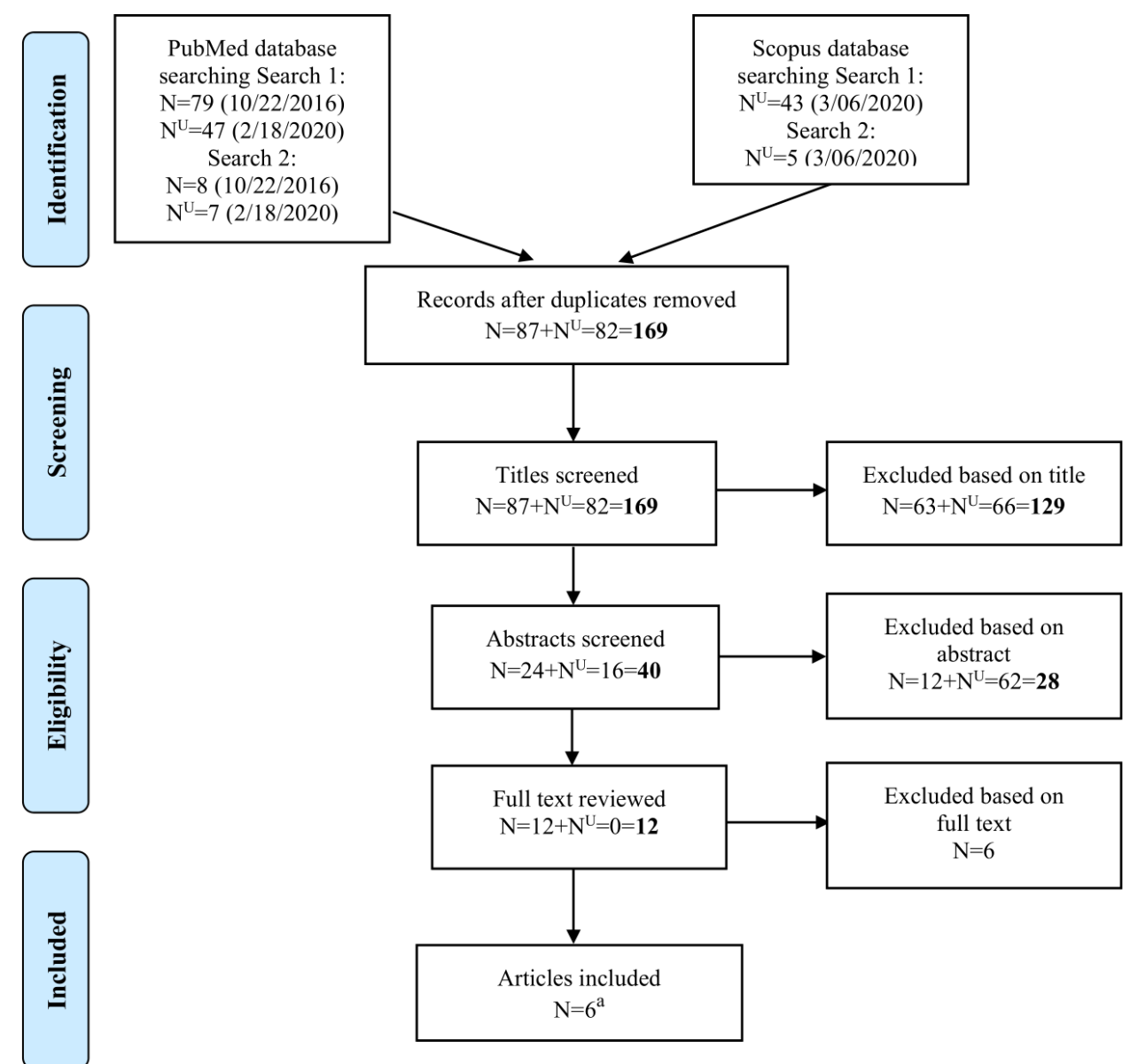

Figure 2 A summary of secondary literature original and updated systematic search selection process. N=The number of potentially qualifying articles from the original search. $\mathrm{N}^{\mathrm{U}}=$ The number of potentially qualifying articles from the updated search. ${ }^{a}$ All six qualifying meta-analyse ${ }^{47-52}$ also emerged as qualifying from the physical activity guidelines for Americans Advisory Committee scientific report original search. ${ }^{1345} 46$

group put on antihypertensive medications as part of the intervention arm (MEDSalone); (4) reported preintervention and postintervention BP for each treatment arm; (5) reported the intensity, time or type of the acute (ie, a single session of exercise) or the frequency, intensity, time or type of the chronic (ie, training) exercise intervention; (6) prior to testing implemented a placebo and medication washout or run-in period and (7) were peerreviewed articles published in English. Trials involving diet modifications in addition to exercise, or populations with chronic disease (s) (eg, cancer, coronary artery disease, HIV/AIDS) were excluded. Acute studies were excluded if their purpose was to measure BP during the exercise intervention and reported $\mathrm{BP} \leq 10 \mathrm{~min}$ into the postexercise intervention recovery period.

\section{Secondary literature search strategy and selection process}

After consultation with a medical librarian (JL), we ran comprehensive Boolean searches in PubMed from 1940 to 22 October 2016 and updated this search from 22 October 2016 to 18 February 2020 in PubMed and from 22 October 2016 to 6 March 2020 in Scopus. See online supplemental SDC 2 for the original and updated search strategy and figure 2 for the selection process.
Included MAs were those that: (1) contained RCTs and/ or controlled trials with a non-exercise, non-diet control group; (2) involved healthy adults $>18$ year with hypertension; (3) investigated the relationship between exercise and BP; (4) reported preintervention and postintervention BP; (5) performed moderator analyses and (6) were peer-reviewed articles published in English or Chinese. Trials involving diet modifications in addition to exercise, populations with chronic disease(s) (eg, cancer, coronary artery disease, HIV/AIDS) or animals were excluded.

We also performed and cross referenced the search in online supplemental SDC 2 with an updated search of the 2018 Physical Activity Guidelines Advisory Committee Scientific Report (PAGACSR) ${ }^{13}$ that was originally run by BBS from 1 January 2006 the end date of the search that was performed for the 2008 PAGACSR to 17 April 2017. We updated this search from 17 April 2017 to 20 February 2020 in PubMed and the Cochrane Library and from 17 April 2017 to 25 February 2020 in CINAHL (online supplemental SDC 3). We also performed manual searches of reference lists of the qualifying MAs to identify additional studies. See online supplemental SDC 3 for a summary of the PAGACSR secondary literature 


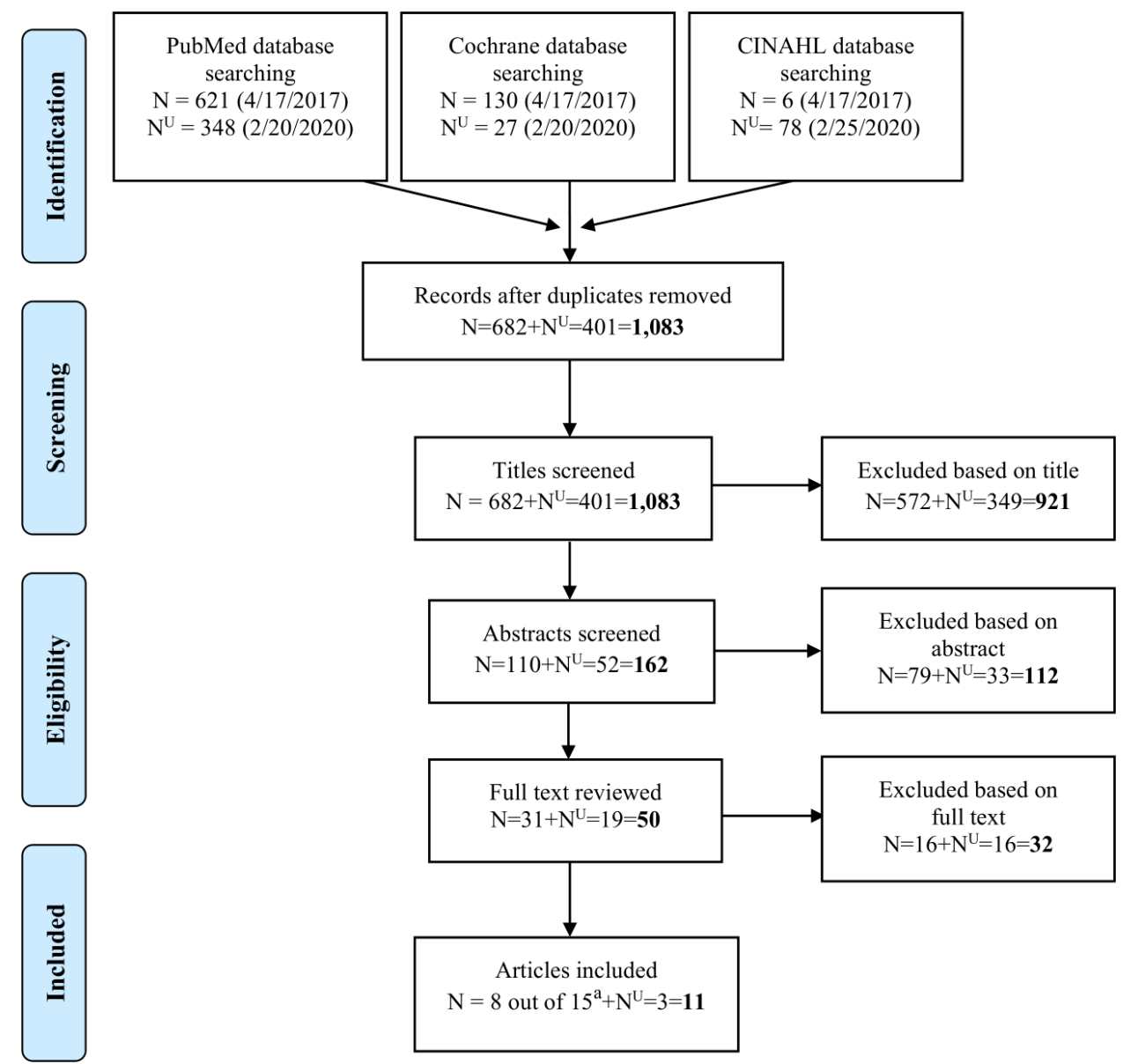

Figure 3 A summary of the original and updated Physical Activity Guidelines Advisory Committee Scientific Report secondary literature systematic search selection process. $\mathrm{N}=$ The number of potentially qualifying articles from the original search. $\mathrm{N}^{\mathrm{U}}=$ The number of potentially qualifying articles from the updated search. ${ }^{a} \mathrm{O}$ the 15 meta-analyses qualifying from the original Physical Activity Guidelines for Americans scientific report search, ${ }^{134546}$ eight of these conducted moderator analyses and qualified for this meta-review. ${ }^{47-49} 51-535556$ CINAHL, Cumulative Index to Nursing and Allied Health Literature.

original and updated search strategy and figure 3 for the selection process.

\section{Data extraction}

The titles, abstracts and full text of the identified articles were independently screened by two reviewers. We used the Joint National Committee Seven BP classification scheme for data extraction purposes because the literature searched was based on this BP classification scheme. ${ }^{14}$ For the primary level RCTs, in cases when the preintervention and postintervention $\mathrm{BP} \pm \mathrm{SD}, \mathrm{SE}$, or 95\% CI were not reported, the authors were contacted to obtain these data. If the author did not respond and the data were available in figure form, Web Plot Digitizer V.4.2 released 7 April 2019 (https://automeris.io/ WebPlotDigitizer/) was used to exact these data from the figure. Two abstractors independently extracted data. Disagreements were resolved by discussion.

\section{Risk of bias and methodological study quality assessments}

For the primary level literature, we performed a risk of bias (RoB) assessment with the Cochrane RoB Tool $\mathrm{V} .2^{15}$ for RCTs that were double-blinded ${ }^{16-22}$ or blinded $^{23}$ crossover or double-blinded ${ }^{2425}$ or blinded ${ }^{2627}$ parallel designs. See online supplemental SDC 4 for a summary of the Cochrane RoB assessment of the RCTs. The RoB V.2 domains assessed were the randomisation process, deviation from intended interventions, missing outcome data, measurement of the outcomes and selection of reported results. For a study to exhibit a low RoB, all domains were judged to be of low RoB; for a study to exhibit some concerns, at least one of the domains was judged to have some concerns; and for a study to exhibit a high RoB at least one domain was judged to be of high RoB or multiple domains were judged to have some concerns that substantially lowered the confidence in the findings. Heterogeneity between studies was gauged by the Higgins $\mathrm{I}^{2}$ statistic with values ranging from 0 (homogeneity) to 1.00 (greater heterogeneity) and associated p values. ${ }^{28}$ We also evaluated the potential for publication and other reporting biases for each treatment arm by visually examining the distribution and asymmetry of funnel plots containing the SBP and DBP mean change by the $\mathrm{SE}$ of the mean change for EXalone, MEDSalone and EX+MEDScombined. ${ }^{29}$ 
For the secondary level literature, we performed a methodological study quality assessment using a modified version of the Assessment of Multiple Systematic Reviews (AMSTAR) ${ }^{30}$ containing 18 items that was adapted for exercise related health outcomes such as BP (AMSTARExBP) ${ }^{31}$ See online supplemental SDC 5 for a summary of the AMSTARExBP assessment of the study methodological quality of the MAs. Systematic reviews meeting $\leq 9(50 \%)$ of the items were rated as low, those meeting between 9 and $<13(50 \%-69 \%)$ of the items were rated as moderate, and those meeting $\geq 13(\geq 70 \%)$ were rated as high study methodological quality.

\section{Statistical methods for the primary literature RCTs}

Due to the inherent limitations in the RCTs regarding the sparsity of sample SDs reported and the complexity of the NMA model that was needed, there was no statistical software package available to perform direct treatment arm comparisons of the BP changes from EXalone, MEDSalone, and EX+MEDScombined. Therefore, we developed the statistical model described below to perform an NMA to answer the question: Do the BP effects of exercise and medication combined add up to the BP effects of the sum of their parts among adults with hypertension?

We assumed that there was a total of $K$ RCTs, and within each RCT there were up to three treatment arms $j=1,2,3$, which denoted EXalone, MEDSalone, and EX+MEDScombined, respectively. For our NMA data, the values of $K$ were: 7 for SBP Acute; 6 for DBP Acute; 5 for both SBP and DBP Chronic; 12 for SBP Total and 11 for DBP total as reported in table 1 . We then let $n_{k j}, Y_{k j}$, and $S_{k j}$ denote the sample size, SBP and DBP change, and sample SD from each $j$ th treatment arm in the $k$ th RCT for $j=1,2,3$ and $k=1,2, \ldots, K$. Following Yao et $a l^{32} 33$ and Li $e t a l,{ }^{34}$ we assumed the NMA model for $Y_{k j}$ (ie, the SBP or DBP change) as:

Equation (1): $Y_{k j}=\mu_{j}+e^{\phi_{j}} \gamma_{k j}+\varepsilon_{k j}, \quad \varepsilon_{k j} \sim N\left(0, \sigma_{k j}^{2} / n_{k j}\right)$,

where $\mu_{\mathrm{j}}$ and $\sigma_{k j}^{2}$ were the mean and the variance of an individual RCT SBP or DBP change and $e^{\phi_{i}}$ was the SD of the random effect for the $j$ th treatment arm in the $k$ th RCT. Following Li et al, ${ }^{34}$ in Equation (1), a 3-dimensional vector of the random effects $\gamma_{k}=\left(\gamma_{k 1}, \gamma_{k 2}, \gamma_{k 3}\right)^{\prime}$ was assumed to follow a 3-dimensional normal distribution with the mean $(0,0,0)^{\prime}$ and a $3 \times 3$ correlation matrix p. Following Lu and Ades, ${ }^{35} 36$ we assumed the correlation matrix $\boldsymbol{\rho}$ was specified to reflect small to moderate correlations. As discussed in Gwon et al, ${ }^{37}$ the formulation $e^{\phi_{j}} \gamma_{k j}$ in equation (1) greatly improved convergence of the SAS PROC NLMIXED. Within the $k$ th RCT, if $S_{k j}$ (ie, the sample SD) was available, following Yao et al, ${ }^{32} 33$ we further assumed:

$$
\text { Equation (2): } \frac{\left(n_{k j}-1\right) s_{k j}^{2}}{\sigma_{k j}^{2}} \sim \chi_{n_{k j-1}}^{2},
$$

where $\chi_{n_{k j-1}}^{2}$ denoted the $\chi^{2}$ distribution with $\left(n_{k j}-1\right)$ degrees of freedom. For those RCTs in which $S_{k j}$ was not available, we assumed $\sigma_{k j}^{2}=\sigma_{k^{*} j}^{2}$, where $k^{*}$ is the RCT that had the largest sample SD. Under the above NMA model, the mean SBP and DBP changes corresponding to $\mathrm{EX}_{\text {alone }}$ vs EX+MEDS combined MEDS $_{\text {alone }}$ vs EX+MEDS $\mathrm{EX}_{\text {alone }}+\mathrm{MEDS}_{\text {alone }}$ vs EX+MEDS ${ }_{\text {combined, }}$ and $\mathrm{EX}_{\text {alone }}$ vs MEDS $_{\text {alone }}$ were denoted as: $\mu_{1}-\mu_{3}, \mu_{2}-\mu_{3}, \mu_{1}+\mu_{2}-\mu_{3}$, and $\mu_{1}-\mu_{2}$, respectively.

We developed SAS Macro BPNMA that is available to download from http://merlot.stat.uconn.edu/packages/metapack/resources/sas-macros/ to implement the above NMA model in SAS PROC NLMIXED. Our SAS Macro BPNMA automatically calculates the pooled mean SBP or DBP change (Mean), its SE, the df associated with the t-test statistics, and the two-sided $p$ value for each treatment or between treatment arm comparison. For our NMA data, the df ranged from 2 to 9 for each treatment or between treatment arm comparison. Due to the complexity of the NMA model and the sparsity of the NMA RCT data, the pooled SD for each treatment arm was not available or not well defined. For this reason, to account for different $\mathrm{df}$ of the t-test statistic, we calculated the effect size (ES) as follows:

Equation (3): $E S=\Phi^{-1}\left(F_{t v}\left(\frac{\text { Mean }}{S E}\right)\right)$,

where $F_{t_{v}}$ denoted the cumulative distribution function of the t distribution with $v$ degrees of freedom and $\Phi^{-1}$ was the inverse of the standard normal distribution function. The ES defined in equation (3) was unified within the same standard normal scale which facilitated the interpretation and comparison between the different treatment arms that were estimated using the NMA data with different sample sizes. The ES in equation (3) also had a one-to-one correspondence with the $\mathrm{p}$ value for grading the strength of the BP effect as an ES absolute value of:

$<1.64$ insufficient; $1.64-1.96$ small; $1.96-2.58$ moderate; 2.58-3.29 large; and $>3.29$ very large, which corresponded to $\mathrm{p}$ values of: $>0.1 ; \mathrm{p}<0.1-0.05 ; \mathrm{p}<0.05-0.01$; $\mathrm{p}<0.01-0.001$ and $\mathrm{p}<0.001$, respectively.

The SBP or DBP change and its corresponding $95 \%$ CI from a RCT together with the overall estimated treatment effect and its $95 \%$ CI were plotted using R. ${ }^{38}$ The funnel plots $^{3940}$ were produced using $\mathrm{R}$ package metafor ${ }^{41}$ to visionally inspect the publication bias for EXalone, MEDSalone, and EX+MEDScombined, separately, in the total study sample. Finally, R package emdbook ${ }^{42} 43$ was used to calculate $\mathrm{p}$ values of the $\mathrm{I}^{2}$ statistics.

\section{Definitions used for the comparison of the treatment arm BP effects}

The primary level RCTs direct comparisons made between EX+MEDScombined and EXalone, and/or MEDSalone, depending on the result, could be assigned the label—synergistic, additive or neutral. ${ }^{44}$ To be synergistic, the magnitude of the BP reductions achieved by EX+MEDScombined were greater than EXalone plus MEDSalone or greater than the sum of their parts. To be additive, the magnitude of the BP reductions achieved by EX+MEDScombined were equal to EXalone plus MEDSalone or equal to the sum of their parts. To be neutral, the magnitude of the BP reductions achieved by EX+MEDScombined were equal to EXalone or MEDSalone or no 


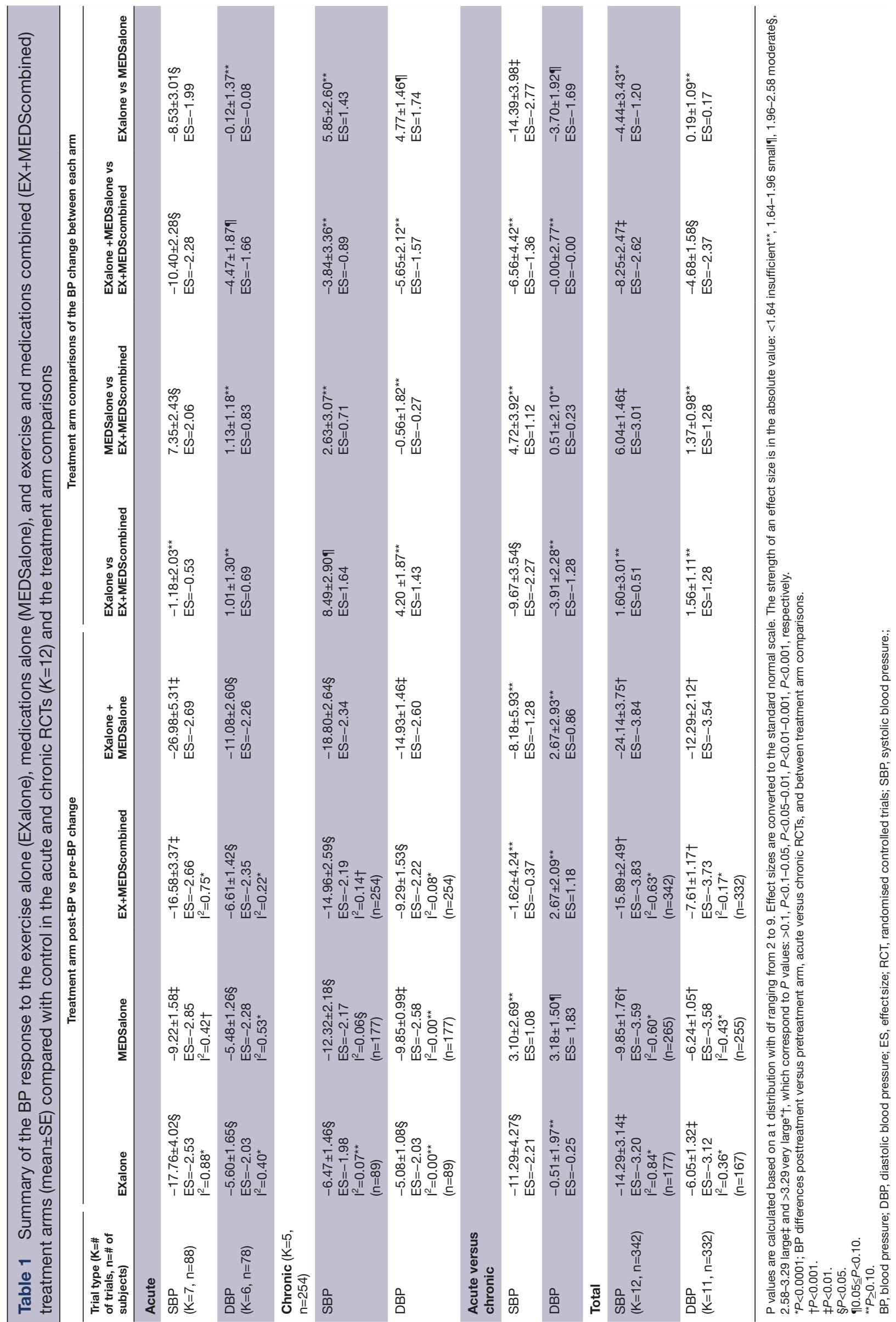


added benefit (or harm). In addition, if the BP effects between EX+MEDScombined were greater than EXalone or MEDSalone, the BP effects of EX+MEDScombined were considered to potentiate the antihypertensive effects of either alone. For the MA moderator analyses, if the authors did not disclose whether medication was a significant moderator or not, we considered the BP effects of EX+MEDScombined compared with EXalone and/or MEDSalone to be neutral or no added benefit (or harm).

\section{The grading of the evidence}

For both literatures, we used the grading system of the 2018 PAGACSR and graded the evidence as strong, moderate, limited or 'not assignable' based on grading criteria that included applicability, generalisability, RoB/ study limitations, quantity and consistency of results across studies, and magnitude and precision of the BP effect. More detailed information about the grading of evidence rubric we used can be found in the 2018 PAGAC scientific report ${ }^{13} 4546$

\section{RESULTS}

\section{The primary literature}

To maximise coverage of the literature we conducted two searches. The first was run in PubMed, Scopus, CINAHL, SPORTDiscus and the Cochrane Library to 21 July 2016 and updated in PubMed and Scopus on 26 February 2020 yielding 33039 potentially qualifying trials (online supplemental SDC 1, figure 1). Of these, 12 RCTs qualified. ${ }^{16-27}$

\section{Study and sample characteristics}

Online supplemental SDC 6 contains the descriptions of the sample, study and intervention characteristics of the seven qualifying acute and online supplemental SDC 7 the five chronic RCTs. There was a total of 342 subjects $(\mathrm{n}=88$ in the acute RCTs; $\mathrm{n}=254$ in the chronic RCTs) with a mean of $28.5 \pm 32.2$ subjects per RCT ranging from 8 to 120 subjects. Participants were mostly physically inactive, middle-aged to older adults with an untreated resting SBP/DBP of $149.3 \pm 11.6 / 93.4 \pm 7.7 \mathrm{~mm}$ $\mathrm{Hg}$. A history of antihypertensive treatment was disclosed in seven RCTs ( $n=5$ acute; $n=2$ chronic). Of these, two contained a mix of treated and untreated subjects, ${ }^{16} 24$ two untreated subjects ${ }^{1726}$ and three treated subjects. ${ }^{20-22}$ Prior to testing, all investigators implemented a placebo and medication washout or run-in period that averaged $3.4 \pm 2.0$ week. When reported, $94 \%$ of the subjects in the acute RCTs were men, $74 \%$ of the subjects in the chronic RCTs were women and $60 \%$ of the subjects in the total sample were women.

Of the 12 qualifying RCTs, seven were acute doubleblinded cross-over designs, ${ }^{16-22}$ one was a chronic blinded cross-over design ${ }^{23}$ two were chronic double-blinded parallel designs ${ }^{24} 25$ and two were blinded parallel designs. ${ }^{26}{ }^{27}$ Five of the acute RCTs had three treatmentsEXalone, MEDSalone, and EX+MEDScombined, ${ }^{17}$ 19-22 while Wilcox et $a l^{16}$ and Cléroux et $a l^{18}$ had two treatments- EXalone and EX+MEDScombined. Only one of the chronic interventions had three treatments, ${ }^{23}$ while Kelemen $e t a l^{24}$ and Ohta $e t a l^{26}$ had EXalone and EX+MEDScombined, and Ammar ${ }^{27}$ and Maruf et $a l^{25}$ had MEDSalone and EX+MEDScombined. The antihypertensive medications examined included beta-blockers ( $K=4$, two acute and two chronic RCTs), calcium channel blockers ( $K=3$, one acute and two chronic RCTs), angiotensin converting enzyme inhibitors $(K=3$, two acute and one chronic RCTs), angiotensin I or II receptor blockers $(K=3$, two acute and one chronic RCTs) and diuretics ( $K=1$, chronic RCT).

The acute exercise interventions were primarily aerobic $^{16-1921} 22$ except Queiroz et $a l^{20}$ which involved dynamic resistance exercise and were of moderate to vigorous intensity for $38.8 \pm 7.6 \mathrm{~min}$ per session. The chronic exercise interventions were also mostly aerobic $^{2325-27}$ except for Kelemen $e t a l^{24}$ which involved concurrent exercise training and were of moderate to vigorous intensity performed 3 day/week for $37.0 \pm 15.7 \mathrm{~min}$ per day for $9.8 \pm 3.5$ week. All investigators measured BP preintervention and postintervention by auscultation in the laboratory ${ }^{16-22} 24-27$ except Radaelli $e t$ $a l^{23}$ who reported ambulatory BP only.

The BP response to exercise alone (EXalone), medications alone (MEDSalone), and exercise and medications combined (EX+MEDScombined)

A summary of the $\mathrm{BP}$ response $($ mean $\pm \mathrm{SE})$ to the three treatments compared with control is displayed in the left side of table 1. Furthermore, forest plots of the BP response to the three treatments compared with control are in online supplemental SDC 8 for EXalone, online supplemental SDC 9 for MEDSalone and online supplemental SDC 10 for EX+MEDScombined Among the total sample, SBP and DBP were significantly reduced postintervention vs preintervention in each treatment arm by $10-16 \mathrm{~mm} \mathrm{Hg}$ for SBP and $6-8 \mathrm{~mm} \mathrm{Hg}$ for DBP $(P s<0.01, P s<0.001)$, with a large strength of SBP and DBP effect for EXalone and very large strength of SBP and DBP effect for MEDSalone and EX+MEDScombined. In the acute RCTs, the BP reductions postintervention versus preintervention in the three treatments compared with control were 9-18 $\mathrm{mm} \mathrm{Hg}$ for $\mathrm{SBP}$ and 6-7 $\mathrm{mm} \mathrm{Hg}$ for DBP $(0.05 \leq P s<0.10)$, with a moderate strength of SBP and DBP effect for EXalone and a moderate strength of DBP effect and large strength of SBP effect for MEDSalone and EX+MEDScombined. In the chronic RCTs the $\mathrm{BP}$ reductions were $7-15 \mathrm{~mm} \mathrm{Hg}$ for SBP and $5-10 \mathrm{~mm}$ $\mathrm{Hg}$ for DBP $(0.05 \leq P s<0.10)$, with a moderate strength of SBP and DBP effect for EXalone and EX+MEDScombined and a moderate strength of SBP and large strength of DBP effect for MEDSalone. The SBP reductions for EXalone were greater in the acute than chronic RCTs by $11 \mathrm{~mm} \mathrm{Hg}(P<0.05)$ with a moderate strength of SBP effect; whereas the DBP reductions for MEDSalone were greater in the chronic than the acute RCTs by $3 \mathrm{~mm} \mathrm{Hg}$ 
$(0.05 \leq P<0.10)$, with a small strength of DBP effect. There were no differences in the DBP reductions for EXalone, SBP reductions for MEDSalone, and the SBP and DBP reductions for $\mathrm{EX}+\mathrm{MEDScombined} \mathrm{between} \mathrm{the} \mathrm{acute}$ and chronic RCTs (Ps>0.05).

\section{Risk of bias}

Using the Cochrane RoB tool V.2, ${ }^{15}$ we found most of the RCTs exhibited high levels of RoB ( $k=10$, 83.3\%), with $83.3 \%$ exhibiting some concerns primarily for missing measurement outcomes and selection of reported results, and $70 \%$ exhibiting some concerns with the randomisation process (online supplemental SDC 4). The I ${ }^{2}$ statistic in table 1 averaged 0.29 and ranged from 0.00 (homogeneity) to 0.88 (greater heterogeneity) with greater heterogeneity among the acute RCTs $(0.53)$ than chronic RCTs (0.06). Visual inspection of the distribution and asymmetry of the funnel plots of the SBP and DBP mean change versus SE are in online supplemental SDC 11 for EXalone, online supplemental SDC 12 for MEDSalone, and online supplemental SDC 13 for EX+MEDScombined. The funnel plots revealed $28 \%-60 \%$ of the studies fell outside of the inverted triangle indicating there was publication bias or other reporting bias and between study heterogeneity for the SBP and DBP responses to EXalone, MEDSalone and EX+MEDScombined.

\section{The treatment arm comparisons of the BP response to exercise} alone (EXalone), medications alone (MEDSalone), and exercise and medications combined (EX+MEDScombined)

A summary of the BP response (mean \pm SE) between the treatment arm comparisons is displayed in the right side of table 1. When comparing EXalone to EX+MEDScombined, the SBP and DBP reductions were not different in the total sample and acute RCTs $(P s \geq 0.10)$. However, in the chronic RCTs, the $\mathrm{SBP}$ reductions were greater by $8 \mathrm{~mm} \mathrm{Hg}$ for $\mathrm{EX}+\mathrm{MEDScom}-$ bined than EXalone $(P<0.05, P<0.10)$, with a small strength of SBP effect; while the DBP reductions were not different for EXalone vs EX+MEDScombined $(P \geq 0.10)$. The SBP reductions were $10 \mathrm{~mm} \mathrm{Hg}$ greater for EXalone vs EX+MEDScombined in the acute than the chronic RCTs $(P<0.05)$, with a moderate strength of SBP effect; whereas the DBP reductions for EXalone vs EX+MEDScombined were not different between the acute and chronic RCTs $(P \geq 0.10)$. These findings reveal no BP benefit of $\mathrm{EX}+\mathrm{MEDScombined} \mathrm{over}$ EXalone except for perhaps a small advantage of EX+MEDScombined over EXalone for SBP in the chronic RCTs.

When comparing MEDSalone to EX+MEDScombined, the SBP reductions in the total sample were greater by $6 \mathrm{~mm} \mathrm{Hg}(P<0.01)$ and acute RCTs by $7 \mathrm{~mm} \mathrm{Hg}(P<0.05)$ for $\mathrm{EX}+\mathrm{MEDScombined} \mathrm{than} \mathrm{MEDSalone} \mathrm{with} \mathrm{a} \mathrm{large} \mathrm{and}$ moderate strength of SBP effect, respectively; whereas the DBP reductions were not different in the total sample and acute and chronic RCTs for MEDSalone vs EX+MEDScombined $(P s \geq 0.10)$. Furthermore, the SBP and DBP reductions for MEDSalone vs EX+MEDScombined were not different between the acute and chronic RCTs $(P s>0.05)$. These findings reveal an advantage of EX+MEDScombined over MEDSalone for SBP but not DBP.

When comparing EXalone plus MEDSalone to EX+MEDScombined in the total sample and acute RCTs, the SBP $(P s<0.05)$ and DBP $(0.05 \leq P s<0.10)$ reductions were greater for EXalone plus MEDSalone vs EX+MEDScombined by 8-10 $\mathrm{mm} \mathrm{Hg}$ for SBP and $5 \mathrm{~mm} \mathrm{Hg}$ for DBP, with a moderate to large strength of SBP effect and a small to moderate strength of DBP effect. In contrast, the SBP and DBP reductions were not different for EXalone plus MEDSalone vs EX+MEDScombined in the chronic RCTs or between the acute and chronic RCTS $(P \mathrm{~s}>0.10)$. Therefore, in the total sample the SBP and DBP effects of EX+MEDScombined were not additive nor synergistic, a result largely driven by the acute RCTs.

When comparing the EXalone to MEDSalone in the total sample, the SBP and DBP reductions were not different for EXalone than MEDSalone $\left(P_{S}>0.10\right)$. However, in the acute RCTs, the SBP reductions were greater by $8 \mathrm{~mm} \mathrm{Hg}$ for EXalone than MEDSalone $(P<0.05)$, with a moderate strength of SBP effect, but the DBP reductions were not different for EXalone than MEDSalone (P>0.10); while in the chronic RCTs the DBP reductions were greater for MEDSalone vs EXalone by $5 \mathrm{~mm} \mathrm{Hg}(0.05 \leq P<0.10)$, with a small strength of DBP effect, but the SBP reductions were not different for EXalone than MEDSalone $(P \geq 0.10)$. Furthermore, the SBP reductions were greater by $14 \mathrm{~mm} \mathrm{Hg}$ $(P \geq 0.01)$ and DBP by $4 \mathrm{~mm} \mathrm{Hg}(0.05 \leq P<0.10)$ for EXalone vs MEDSalone between the acute than chronic RCTs, with a large strength of SBP effect and small strength of DBP effect, respectively. In the total sample, EXalone is equally effective as MEDSalone in lowering BP; however, EXalone is moderately more effective than MEDSalone in lowering SBP in the acute RCTs and MEDSalone elicits a small advantage in lowering DBP in the chronic RCTs.

In summary, in the total sample, there is a large to very large strength of BP effect for EXalone, MEDSalone, and EX+MEDScombined eliciting SBP reductions ranging from 10 to $16 \mathrm{~mm} \mathrm{Hg}$ and DBP ranging from 6 to $8 \mathrm{~mm} \mathrm{Hg}$. When directly comparing the $\mathrm{BP}$ reductions from EXalone and/ or MEDSalone to EX+MEDScombined for the total sample: (1) EXalone and EX+MEDScombined appear equally effective in lowering SBP and DBP, noting the exception of SBP in the acute RCTs in which EX+MEDScombined conferred a small advantage over EXalone; (2) EX+MEDScombined is more effective than MEDSalone in lowering SBP, largely driven by the acute RCTs, but appears equally as effective as MEDSalone in lowering DBP; (3) EXalone plus MEDSalone elicits greater SBP and DBP reductions than EX+MEDScombined, largely driven by the acute RCTs; and (4) EXalone and MEDSalone appear equally effective in lowering SBP and DBP, noting the exception of DBP in the chronic RCTs in which MEDSalone conferred a small advantage over EXalone. Now to answer the question-the BP reductions of EX+MEDScombined do not add up to the sum of the BP reductions of each treatment alone indicating they are not additive nor synergistic. Yet, when directly comparing the three treatment arms, EXalone appears equally as effective as 
MEDSalone and EX+MEDScombined in lowering SBP and DBP. Furthermore, EX+MEDScombined is more effective than MEDSalone in lowering SBP, while both appear equally effective in lowering DBP.

\section{Grading of the evidence in the primary literature}

Strong evidence demonstrates EXalone, MEDSalone, and exercise and medication combined reduce $\mathrm{BP}$ among adults with hypertension. Grade: Strong.

Strong evidence demonstrates exercise and medication combined elicit BP reductions of less magnitude than the sum of EXalone plus MEDSalone among adults with hypertension. Grade: Strong.

Strong evidence demonstrates exercise and medication combined potentiate the magnitude of the SBP reductions from medications alone among adults with hypertension. Grade: Strong.

Insufficient evidence is available to determine whether exercise and medication combined potentiate the DBP reductions from medicine alone among adults with hypertension. Grade: not assignable.

Limited evidence suggests exercise and medication combined potentiate the SBP reductions from EXalone among adults with hypertension. Grade: limited.

Insufficient evidence is available to determine whether exercise and medication combined potentiate the DBP reductions from EXalone among adults with hypertension. Grade: not Assignable.

Limited evidence suggests EXalone elicits greater SBP reductions than MEDSalone among adults with hypertension. Grade: limited.

Limited evidence suggests MEDSalone elicits greater DBP reductions than EXalone among adults with hypertension. Grade: limited.

\section{The secondary literature}

To maximise coverage of the secondary literature we conducted two searches. The first was run in PubMed to 22 October 2016 and updated in PubMed on 18 February 2020 and Scopus from 22 October 2016 to 6 March 2020 and yielded 182 potentially qualifying trials (online supplemental SDC 2, figure 2). Of these, a total of six MAs of which four involved RCTs ${ }^{4-50}$ and two controlled trials ${ }^{5152}$ qualified. This search was cross referenced with the PAGACSR original search that was run from 1 January 2006 to 7 April 2017 and updated on 20 February 2020 in PubMed and the Cochrane Library, and updated on 25 February 2020 in the CINAHL databases. The PAGACSR original and updated searches yielded 1210 potentially qualifying trials (online supplemental SDC 3, figure 3). Of these, eight MAs of RCTs ${ }^{47-50} 53-56$ and three of controlled trials ${ }^{515257}$ qualified, and they included the six qualifying $\mathrm{MAs}^{47-52}$ from the online supplemental SDC 2 search. In addition, we performed manual searches of the references lists within the MAs and examined electronic journal table of contents and medical search engines routinely sent to the investigators related to exercise and hypertension from which two additional MAs were identified, one of which included RCTs $^{58}$ and one RCTs and controlled trial. ${ }^{59}$

\section{Study and sample characteristics}

See online supplemental SDC 14 for the description of the sample, study and intervention characteristics categorised by exercise modality of the 13 qualifying MAs of which nine were MAs of RCTs ${ }^{4-50} 53-5658$ and 4 MAs of RCTs and controlled trials ${ }^{51525759}$ with a range of 8-93 trials per MA. Two MAs involved aerobic exercise training, one acute and two chronic (ie, training) dynamic resistance exercise, one chronic aerobic and resistance combined (ie, concurrent), and seven chronic complementary and alternative types of exercise that included Daduanjin, Qigong, Tai Chi, Wuqinxi, Yijinjing and Yoga. The sample size was 28468 adults $>18$ year, ranging from 572 to 5223 participants. When the data were provided, the samples were generally an equal mix of men and women, and mostly Caucasian or Asian. The overall study methodological study quality of the literature was high, with 54\% scoring moderate and $46 \%$ high on the AMSTARExBP (online supplemental SDC 5).

Evidence on the influence of antihypertensive medication on the $\mathrm{BP}$ response to exercise

Of the 13 qualifying MAs, six did not report if medication was a significant moderator or not, ${ }^{4745} 52535759$ one reported medication was not a significant moderator ${ }^{54}$ and six found medication was a significant moderator of the $\mathrm{BP}$ response to exercise. ${ }^{450515556}$

MacDonald et $a l^{\tilde{1}}$ reported moderate intensity, dynamic resistance training performed on average 3 day/week for 14 week lowered SBP $5.7 \mathrm{~mm} \mathrm{Hg}(-9.0$ to -2.7$)$ and DBP $5.2 \mathrm{~mm} \mathrm{Hg}(-8.4$ to -1.9$)$ among 667 adults with hypertension ( $\sim 28 \%$ of the total sample). In addition, $15 \%$ of the total sample $(\mathrm{n}=349)$ was on medication, but $32 \%$ of the trails did not disclose information on medication use. Greater SBP/DBP reductions occurred among samples exercising and not taking medication $(-4.3 \mathrm{~mm}$ $\mathrm{Hg}(-6.2$ to -2.2$) /-3.5 \mathrm{~mm} \mathrm{Hg}(-5.0$ to -2.1$))$ than those exercising and taking medication $(-0.4 \mathrm{~mm} \mathrm{Hg}(-3.8$, $3.0) /-1.2 \mathrm{~mm} \mathrm{Hg}(-3.4,1.0))$ suggesting the BP benefits of EXalone were greater than those of EX+MEDScombined. However, the authors urged caution in interpreting these findings because they pertained to the total sample and not to adults with hypertension per se, there was a small percentage of the sample taking medications, and a general lack of disclosure of this information.

Jin et a $\tilde{l}^{\tilde{0}}$ examined the BP benefits of traditional Chinese exercise that included Daduanjin, Qigong, Tai Chi, Wuqinxi, Yijinjing and Yoga among 1164 adults with hypertension. These authors did not report the overall BP reductions of their sample. However, their subgroup analyses revealed traditional Chinese exercise combined with medication lowered SBP $-13.9 \mathrm{~mm} \mathrm{Hg}(-16.3,-10.1)$ and DBP $-4.9 \mathrm{~mm} \mathrm{Hg}(-4.9$ to -4.7$)$ more than MEDSalone. Similarly, Xiong et al found greater SBP/DBP reductions from Baduanjin combined with medication than 
MEDSalone (-7.5 mm Hg (-11.4,-3.6) / -3.6 mm Hg (-5.2 to -1.8$)$ ) and Qigong combined with medication than Qigong EXalone $(-12.0 \mathrm{~mm} \mathrm{Hg}(-15.6,-8.5) /-5.3 \mathrm{~mm}$ $\mathrm{Hg}(-8.1$ to -2.4$))^{48} 56$; while Wang et al found greater SBP/DBP reductions with Tai Chi combined with medication than Tai Chi EXalone $(-9.3 \mathrm{~mm} \mathrm{Hg}(-10.9$ to -7.8$) /-7.2 \mathrm{~mm} \mathrm{Hg}(-7.7$ to -6.6$)) .^{55}$ Furthermore, Xiong et al found Qigong EXalone $(-7.9 \mathrm{~mm} \mathrm{Hg}(-16.8$, 1.0)/ $-6.1 \mathrm{~mm} \mathrm{Hg}(-9.6,-2.6)),{ }^{56}$ Zhong et al found Tai Chi EXalone $(-9.0 \mathrm{~mm} \mathrm{Hg}(-14.0,-4.1) /-5.6 \mathrm{~mm} \mathrm{Hg}$ $(-8.8$ to -2.4$)),{ }^{58}$ and Wang et a $\tilde{l}^{5}$ found Tai Chi EXalone $(-14.3 \mathrm{~mm} \mathrm{Hg}(-14.31,-14.29) /-6.0 \mathrm{~mm} \mathrm{Hg}(-6.01$ to -5.99$)$ ) elicited greater SBP/DBP reductions than MEDSalone. Collectively, the findings of Jin $e t a l,{ }^{50}$ Xiong et $a l^{4856}$ and Wang et a ${ }^{55}$ indicated that complementary and alternative forms of EX+MEDScombined elicited greater BP reductions than MEDSalone. In addition, the findings of Xiong et $a l,{ }^{56}$ Zhong et $a l,{ }^{58}$ and Wang et $a b^{55}$ demonstrated that complementary and alternative forms of EXalone resulted in greater BP reductions than MEDSalone.

In summary, six MAs did not report the results of their moderator analyses involving medication ${ }^{47} 4952535759$ and one $^{54}$ found medication was not a significant moderator of the BP response to exercise suggesting EX+MEDScombined added no BP benefit to EXalone, findings that are consistent with those of Naci $e t a l^{9}$ and Noone $e t a l .{ }^{10}$ One MA found EX+MEDScombined attenuated the BP benefits of EXalone ${ }_{(51)}$. Four MAs found greater BP reductions with EX+MEDScombined than MEDSalone, ${ }^{48} 505556$ and three MAs found EXalone elicited greater BP reductions than MEDSalone. ${ }^{55568}$

The grading of the evidence of the secondary literature

Strong evidence demonstrates EXalone, MEDSalone, and exercise and medication combined reduce BP among adults with hypertension. Grade: strong.

Strong evidence demonstrates exercise and medication combined potentiate the magnitude of the BP reductions from MEDSalone among adults with hypertension. Grade: strong.

Moderate evidence indicates EXalone elicits greater BP reductions than MEDSalone among adults with hypertension. Grade: moderate.

Insufficient evidence is available to determine whether the exercise and medication combined potentiate the BP reductions from EXalone among adults with hypertension. Grade: not assignable.

\section{DISCUSSION}

The answer to the question: do the combined BP effects of exercise and antihypertensive medications add up to the sum of their parts?

We have conducted the first evidenced-based, comprehensive systematic NMA and meta-review on two literature levels to compare the effectiveness of EX+MEDScombined to EXalone and/or MEDSalone, on BP among adults with hypertension. The first search involved primary level RCTs that isolated and directly compared EX+MEDScombined to EXalone and/or MEDSalone, and the second involved MAs of RCTs and controlled trials on exercise and BP that performed moderator analyses. A summary of the grading of the evidencebased conclusion statements on the BP response to the EXalone, MEDSalone, and EX+MEDScombined is shown in table 2.

In total, there were eight conclusion statements made from the primary literature of which three were strong, three limited, and two not assignable; and four from the secondary literature of which three were strong, one moderate and one not assignable. Strong evidence from the RCTs (online supplemental SDC 6 and 7 and table 2) and MAs (online supplemental SDC 14 and table 2) demonstrates EXalone, MEDSalone, and EX+MEDScombined reduce BP by $2-18 \mathrm{~mm} \mathrm{Hg}$ among adults with hypertension. Strong evidence from the RCTs demonstrates the combined BP effects of exercise and medications are less than the sum of either alone by $5-8 \mathrm{~mm} \mathrm{Hg}$. Despite finding the combined BP effects of exercise and medication are not additive or synergistic, strong evidence from the RCTs indicates that when combined, they potentiate the SBP effect of MEDSalone by $6 \mathrm{~mm} \mathrm{Hg}$ with traditional types of exercise (ie, aerobic and resistance); whereas there was insufficient evidence to make any conclusions about DBP. In addition, there was strong evidence from the MAs that EX+MEDScombined potentiate the SBP and DBP effects of MEDSalone up to $14 \mathrm{~mm} \mathrm{Hg}$ with alternative types of exercise (ie, Daduanjin, Qigong, Tai Chi, Wuqinxi, Yijinjing and Yoga). The evidence from the RCTs and MAs was limited to not assignable, and thus, only suggestive that EX+MEDScombinedmay potentiate the BP reductions from EXalone among adults with hypertension. The evidence of the comparative effects of EXalone and MEDSalone was mixed with limited evidence from the acute RCTs indicating EXalone elicits greater SBP reductions than MEDSalone by $9 \mathrm{~mm} \mathrm{Hg}$; whereas in the chronic RCTs MEDSalone elicits greater DBP reductions than EXalone by $5 \mathrm{~mm} \mathrm{Hg}$; whereas for the MAs moderate evidence indicated that alternative forms of EXalone elicits greater $\mathrm{BP}$ reductions than MEDSalone by $6-9 \mathrm{~mm} \mathrm{Hg}$.

The benefits of lowering BP among those with hypertension are undisputable, however, the methods by which the $\mathrm{BP}$ reductions are achieved are a topic of debate regarding their comparative effectiveness. ${ }^{9}{ }^{10}{ }^{60-63}$ We found EXalone, MEDSalone and EX+MEDScombined all reduced $\mathrm{BP}$ by $2-17 \mathrm{~mm} \mathrm{Hg}$. BP reductions of $5-10 \mathrm{~mm}$ $\mathrm{Hg}$ decrease the relative risk for all-cause mortality $11 \%$, cardiovascular morality $16 \%$, coronary heart disease $18 \%$, and stroke $36 \%$ substantiating the importance of each of these approaches to treat hypertension. ${ }^{3}$ For the first time, we have conducted an NMA of RCTs that isolated the BP effects of EX+MEDScombined to EXalone and/ or MEDSalone (online supplemental SDC 6 and 7). In this way, we were able to deliver strong evidence that the BP effects of exercise and medication combined are not 
Table 2 Summary of the grading of the evidence for the conclusion statements on the blood pressure (BP) response to the exercise alone, medications alone, and exercise and medications combined and their comparisons among adults with hypertension

\section{Conclusion statement}

\section{Grade}

\section{Primary literature*}

Strong evidence demonstrates exercise alone, medication alone and exercise and medication combined reduce Strong $^{16-27}$ BP among adults with hypertension.

$\begin{array}{lc}\text { Strong evidence demonstrates exercise and medication combined elicit BP reductions of less magnitude than } & \text { Strong } \\ \text { the sum of exercise alone plus medication alone among adults with hypertension. } & \text { Strong }{ }^{16-27} \\ \begin{array}{l}\text { Strong evidence demonstrates exercise and medication combined potentiate the magnitude of the SBP } \\ \text { reductions from medication alone among adults with hypertension. }\end{array} & \end{array}$

Insufficient evidence is available to determine whether exercise and medication combined potentiate the DBP
reductions from medicine alone among adults with hypertension.

Limited evidence suggests exercise and medication combined potentiate the SBP reductions from exercise Limited ${ }^{16-2426}$ alone among adults with hypertension.

\section{Insufficient evidence is available to determine whether exercise and medication combined potentiate the DBP Not assignable ${ }^{16-2426}$} reductions from exercise alone among adults with hypertension.

Limited evidence suggests exercise alone elicits greater SBP reductions than medication alone among adults $\quad$ Limited $^{16-23}$ with hypertension.

Limited evidence suggests medication alone elicits greater DBP reductions than exercise alone among adults $\quad$ Limited $^{16-23}$
with hypertension.

\section{Secondary literature $\dagger$}

$\begin{array}{lc}\begin{array}{l}\text { Strong evidence demonstrates exercise alone, medication alone, and exercise and medication combined } \\ \text { reduce BP among adults with hypertension. }\end{array} & \text { Strong }{ }^{47-59} \\ \begin{array}{l}\text { Strong evidence demonstrates exercise and medication combined potentiate the magnitude of the BP } \\ \text { reductions from medication alone among adults with hypertension. }\end{array} & \text { Strong } \\ \end{array}$

\section{Moderate evidence indicates exercise alone elicits greater BP reductions than medication alone among adults Moderate ${ }^{55558}$} with hypertension.

Insufficient evidence is available to determine whether the exercise and medication combined potentiate the BP Not assignable. ${ }^{474952535759}$ reductions from exercise alone among adults with hypertension.

\footnotetext{
${ }^{*}$ The conclusions in the primary literature are largely based on evidence from the randomised controlled trials involving acute and chronic aerobic and resistance exercise.

†The conclusions in the secondary literature are largely based on evidence from the meta-analyses involving chronic complementary and alternative types of exercise.

DBP, diastolic BP; SBP, systolic BP.
}

additive to the BP effects of either alone. However, when combined, they potentiate the BP effects of MEDSalone, $\mathrm{BP}$ benefits that appear twice as large for alternative than traditional types of exercise. Furthermore, limited to moderate evidence in the RCTs and MAs, respectively, suggests that EXalone may elicit greater SBP reductions than MEDSalone.

Collectively, our novel findings should temper the widely held belief that antihypertensive medications reduce $\mathrm{BP}$ more than exercise because: alone or combined, they lower BP; when combined they potentiate the BP effects of MEDSalone; and EXalone elicits greater BP reductions than medications alone, although the evidence is stronger for alternative than traditional types of exercise. An important take home message is that when combined, exercise and medication strengthen the BP effects of MEDSalone and should be recommended and implemented as indicated according to existent professional treatment algorithms. ${ }^{163-66}$

\section{Comparison to the literature}

Our findings are in accord with the recent NMA of Naci $e t a l^{9}$ and Noone $e t a l .{ }^{10}$ Despite the efforts of these two investigative teams to compare the effectiveness of EXalone to MEDSalone as antihypertensive therapeutic options, the exercise arms in these NMA contained participants treated with medication so that the $\mathrm{BP}$ effects reported for exercise were not necessarily due to exercise per se, and the medication comparator may have included placebo, usual care, and other medications in addition antihypertensive medications. Naci et $a t^{\theta}$ and Noone $e a^{10}{ }^{10}$ found traditional types of exercise (that were often combined with medications) reduced BP to similar levels as the medication comparator. However, due to the admixture of the treatment comparisons in these NMA, it was not possible to truly isolate the BP effects of EXalone, MEDSalone, and EX+MEDScombined from one another as we have done in this NMA and meta-review.

Reasons why alternative types of exercise when combined with medications potentiate the BP benefits of MEDSalone to levels twice as large as those that result for traditional types of exercise are not clear. A possible explanation is provided by $\mathrm{Wu}$ et al in their MA involving Yoga training among 3517 participants in which they found the greatest BP reductions occurred 
among Yoga interventions that included breathing techniques and meditation and mental relaxation. ${ }^{57}$ Of note, the new International Society of Hypertension Guidelines have added stress reducing measures such as transcendental meditation and Yoga to their recommended lifestyle modifications,${ }^{64}$ and the American College of Sports Medicine has expanded their exercise recommendations to include alternative forms of exercise ${ }^{6768}$ In a more recent MA involving Tai Chi training among 3223 participants, Wu et $a \tilde{p}^{9}$ found the greatest BP reductions occurred among trials published in Chinese than English partially attributed to the lower methodological study quality of the Chinese than English literature. Nonetheless, the MAs from which the treatment comparisons were made in our meta-review were of moderate to high quality. Further research is also warranted to gain insight into why mind body types of exercise appear to confer greater BP benefit than MEDSalone.

\section{Limitations}

Several limitations of this systematic NMA and meta-review should be noted. The RCTs (online supplemental SDC 6 and 7) tended to be older, some involved medications that have become outdated in clinical practice, assumptions were made in our NMA statistical model due to missing data, and they exhibited a high RoB largely due to concerns about the randomisation process and the inability to hide the BP effects of medication from the investigators taking measurements during testing. In addition, the RCTs involved aerobic and resistance exercise and did not disclose ethnicity, while the MAs involved mostly Caucasians and Asians and had a nearly an equal mix of aerobic and resistance exercise and alternative types of exercise. Nearly half of the MAs did not report whether medication was a significant moderator or not. Nonetheless, the strengths are this is a large, evidenced-based comprehensive systematic NMA and meta-review on two literature levels that included RCTs that for the first time isolated the BP effects of each treatment arm being compared in our NMA; and high methodological quality MAs with moderator analyses that compared the treatments arms among adults with hypertension.

\section{CONCLUSIONS}

We have conducted the first large, comprehensive evidenced-based systematic NMA and meta-review adhering to high contemporary standards on two literature levels that compared the effectiveness of EX+MEDScombined to EXalone and/or MEDSalone on BP among adults with hypertension. We found the BP effects of EX+MEDScombined were not additive or synergistic. Yet, the combined effects of exercise and medication bolstered the BP effects of MEDSalone to treat hypertension, and although the evidence was not as robust, EXalone elicited greater BP reductions than medications alone. Another novel finding that merits further investigation is alternative types of exercise appear to exert superior BP benefits than traditional types of exercise. Thus, EXalone, MEDSalone and EX+MEDScombined are all effective antihypertensive therapeutic approaches. However, their combined BP effects are stronger than MEDSalone in the treatment of hypertension.

Acknowledgements The authors gratefully acknowledge the contributions of The 2018 Physical Activity Guidelines Advisory Committee who were involved in the initial search of the secondary literature that included David M. Buchner, Wayne Campbell, Loretta DiPietro, Kirk I. Erickson, Charles H. Hillman, John M. Jakicic, Kathleen F. Janz, Peter T. Katzmarzyk, Abby C. King, William E. Kraus, Richard F. Macko, David X. Marquez, Anne McTiernan, Russell R. Pate, Linda S. Pescatello, Kenneth E. Powell and Melicia C. Whitt-Glover; Anne Brown Rodgers, U.S. Department of Health and Human Services (HHS) consultant for technical writing support; and ICF librarians, abstractors, and HHS support staff. HHS and ICF staff collaborated with the Committee in the design and conduct of the searches by assisting with the development of the analytical frameworks, inclusion/exclusion criteria, and search terms for each primary question; using those parameters, ICF performed the literature searches. The authors also acknowledge Lynette L. Craft, Ph.D., FACSM, Chief Science Officer for the American College of Sports Medicine for her review of the manuscript, and Christina Day, University of Connecticut Department of Kinesiology graduate research assistant for her assistance with data extraction from the figures.

Contributors (1) Concept, design, and data interpretation; (2) drafting of the manuscript and (3) final approval.

Funding The University of Connecticut Centre for Excellence in Teaching and Learning funded YW.

Competing interests None declared.

Patient consent for publication Not required.

Ethics approval Institutional board approval was not required as this NMA and meta-review did not involve data collection with human subjects but involved systematic review of data that had been previously collected.

Provenance and peer review Not commissioned; externally peer reviewed.

Open access This is an open access article distributed in accordance with the Creative Commons Attribution Non Commercial (CC BY-NC 4.0) license, which permits others to distribute, remix, adapt, build upon this work non-commercially, and license their derivative works on different terms, provided the original work is properly cited, appropriate credit is given, any changes made indicated, and the use is non-commercial. See: http://creativecommons.org/licenses/by-nc/4.0/.

\section{ORCID iDs}

Linda S Pescatello http://orcid.org/0000-0002-5841-798X

Yin Wu http://orcid.org/0000-0001-8647-4706

Ming-Hui Chen http://orcid.org/0000-0003-1935-2447

\section{REFERENCES}

1 Whelton PK, Carey RM, Aronow WS, et al. 2017 ACC/AHA/AAPA/ ABC/ACPM/AGS/APhA/ASH/ASPC/NMA/PCNA guideline for the prevention, detection, evaluation, and management of high blood pressure in adults: a report of the American College of Cardiology/ American heart association Task force on clinical practice guidelines. Hypertension 2018;71:e13-115.

2 Virani SS, Alonso A, Benjamin EJ, et al. Heart disease and stroke Statistics-2020 update: a report from the American heart association. Circulation 2020;141:e139-e596.

3 Zanchetti A, Thomopoulos C, Parati G. Randomized controlled trials of blood pressure lowering in hypertension: a critical reappraisal. Circ Res 2015:116:1058-73.

4 Pescatello LS, Buchner DM, Jakicic JM, et al. Physical activity to prevent and treat hypertension: a systematic review. Med Sci Sports Exerc 2019;51:1314-23.

5 Huai P, Xun H, Reilly KH, et al. Physical activity and risk of hypertension: a meta-analysis of prospective cohort studies. Hypertension 2013;62:1021-6. 
6 Liu Y, Shu X-O, Wen W, et al. Association of leisure-time physical activity with total and cause-specific mortality: a pooled analysis of nearly a half million adults in the Asia cohort Consortium. Int $J$ Epidemiol 2018;47:771-9.

7 Liu X, Zhang D, Liu Y, et al. Dose-response association between physical activity and incident hypertension: a systematic review and meta-analysis of cohort studies. Hypertension 2017;69:813-20.

8 Joseph G, Marott JL, Torp-Pedersen C, et al. Dose-response association between level of physical activity and mortality in normal, elevated, and high blood pressure. Hypertension 2019;74:1307-15.

9 Naci H, Salcher-Konrad M, Dias S, et al. How does exercise treatment compare with antihypertensive medications? A network meta-analysis of 391 randomised controlled trials assessing exercise and medication effects on systolic blood pressure. Br J Sports Med 2019;53:859-69.

10 Noone C, Leahy J, Morrissey EC, et al. Comparative efficacy of exercise and anti-hypertensive pharmacological interventions in reducing blood pressure in people with hypertension: a network meta-analysis. Eur J Prev Cardiol 2020;27:247-55.

11 Moher D, Liberati A, Tetzlaff J, et al. Preferred reporting items for systematic reviews and meta-analyses: the PRISMA statement. $J$ Clin Epidemiol 2009;62:1006-12.

12 Moher D, Shamseer L, Clarke M, et al. Preferred reporting items for systematic review and meta-analysis protocols (PRISMA-P) 2015 statement. Syst Rev 2015;4:1-4053-4.

13 U.S. Department of Health and Human Services. Physical activity guidelines for Americans. 2 edn. Washington, DC: U.S. Departmetn of Health and Human Services, 2018.

14 Chobanian AV, Bakris GL, Black HR, et al. Seventh report of the joint National Committee on prevention, detection, evaluation, and treatment of high blood pressure. Hypertension 2003;42:1206-52.

15 Sterne JAC, Savovic J, Page MJ. RoB2: a revised tool for assessing risk of bias in randomized trials. BMJ 2019;366:14898.

16 Wilcox RG, Bennett T, Macdonald IA, et al. The effects of acute or chronic ingestion of propranolol or metoprolol on the physiological responses to prolonged, submaximal exercise in hypertensive men. Br J Clin Pharmacol 1984;17:273-81.

17 Wilcox RG, Bennett T, Macdonald IA, et al. Post-exercise hypotension: the effects of epanolol or atenolol on some hormonal and cardiovascular variables in hypertensive men. Br J Clin Pharmacol 1987;24:151-62

18 Cléroux J, Kouamé N, Nadeau A, et al. Aftereffects of exercise on regional and systemic hemodynamics in hypertension. Hypertension 1992;19:183-91.

19 Beaulieu M, Nadeau A, Lacourcière $Y$, et al. Post-exercise reduction in blood pressure in hypertensive subjects: effects of angiotensin converting enzyme inhibition. Br J Clin Pharmacol 1993;36:331-8.

20 Queiroz ACC, Sousa JCS, Silva ND, et al. Captopril does not potentiate post-exercise hypotension: a randomized crossover study. Int J Sports Med 2017;38:270-7.

21 Ramirez-Jimenez M, Morales-Palomo F, Ortega JF, et al. Effects of intense aerobic exercise and/or antihypertensive medication in individuals with metabolic syndrome. Scand J Med Sci Sports 2018;28:2042-51.

22 Ramirez-Jimenez M, Morales-Palomo F, Ortega JF, et al. Postexercise hypotension produced by supramaximal interval exercise is potentiated by angiotensin receptor blockers. Int J Sports Med 2019;40:756-61.

23 Radaelli A, Piepoli M, Adamopoulos S, et al. Effects of mild physical activity, atenolol and the combination on ambulatory blood pressure in hypertensive subjects. J Hypertens 1992;10:1279-82.

24 Kelemen MH, Effron MB, Valenti SA, et al. Exercise training combined with antihypertensive drug therapy. Effects on lipids, blood pressure, and left ventricular mass. JAMA 1990;263:2766-71.

25 Maruf FA, Akinpelu AO, Salako BL, et al. Effects of aerobic dance training on blood pressure in individuals with uncontrolled hypertension on two antihypertensive drugs: a randomized clinical trial. J Am Soc Hypertens 2016;10:336-45.

26 Ohta M, Tajiri Y, Yamato H, et al. Effects of exercise therapy alone and in combination with a calcium channel blocker or an angiotensin receptor blocker in hypertensive patients. Clin Exp Hypertens 2012;34:523-9.

27 Ammar T. Effects of aerobic exercise on blood pressure and lipids in overweight hypertensive postmenopausal women. J Exerc Rehabil 2015;11:145-50

28 Higgins JPT, Thompson SG, Deeks JJ, et al. Measuring inconsistency in meta-analyses. BMJ 2003;327:557-60.

29 Langan D, Higgins JPT, Gregory W, et al. Graphical augmentations to the funnel plot assess the impact of additional evidence on a meta-analysis. J Clin Epidemiol 2012;65:511-9.
30 Shea BJ, Grimshaw JM, Wells GA, et al. Development of AMSTAR: a measurement tool to assess the methodological quality of systematic reviews. BMC Med Res Methodol 2007;7:10-17.

31 Johnson BT, MacDonald HV, Bruneau ML, et al. Methodological quality of meta-analyses on the blood pressure response to exercise: a review. J Hypertens 2014;32:706-23.

32 Yao H, Chen M-H, Qiu C. Bayesian modeling and inference for metadata with applications in efficacy evaluation of an allergic rhinitis drug. J Biopharm Stat 2011;21:992-1005.

$33 \mathrm{Yao} \mathrm{H}$, Kim S, Chen M-H, et al. Bayesian inference for multivariate meta-regression with a partially observed Within-Study sample covariance matrix. J Am Stat Assoc 2015;110:528-44.

$34 \mathrm{Li} \mathrm{H}$, Chen M-H, Ibrahim JG, et al. Bayesian inference for network meta-regression using multivariate random effects with applications to cholesterol lowering drugs. Biostatistics 2019;20:499-516.

$35 \mathrm{Lu}$ G, Ades AE. Combination of direct and indirect evidence in mixed treatment comparisons. Stat Med 2004;23:3105-24.

36 Lu G, Ades AE. Assessing evidence inconsistency in mixed treatment comparisons. J Am Stat Assoc 2006;101:447-59.

37 Gwon Y, Mo M, Chen Ming-Hui, et al. Network meta-regression for ordinal outcomes: Applications in comparing Crohn's disease treatments. Stat Med 2020;39:1846-70.

38 R Core Team. A language and environment for statistical computing. Vienna, Austria: R Foundation for Statistical Computing, 2020. https://www.R-project.org/

39 Egger M, Davey Smith G, Schneider M, et al. Bias in meta-analysis detected by a simple, graphical test. BMJ 1997;315:629-34.

40 Sterne JA, Egger M. Funnel plots for detecting bias in meta-analysis: guidelines on choice of axis. J Clin Epidemiol 2001;54:1046-55.

41 Viechtbauer W. Conducting meta-analyses in $\mathrm{R}$ with the metafor package. J Stat Software 2010;36:1-48. doi:10.18637/jss.v036.i03

42 Bolker BM. Ecological models and data in R. Princeton, NJ: Princeton University Press, 2008.

43 Bolker B. emdbook: ecological models and data in $R$. R package version 1.3.12, 2020

44 Roell KR, Reif DM, Motsinger-Reif AA. An introduction to terminology and methodology of chemical Synergy-Perspectives from across disciplines. Front Pharmacol 2017;8:1-11.

45 Torres A, Tennant B, Ribeiro-Lucas I, et al. Umbrella and systematic review methodology to support the 2018 physical activity guidelines Advisory Committee. J Phys Act Health 2018;15:805-10.

46 Powell KE, King AC, Buchner DM, et al. The scientific foundation for the physical activity guidelines for Americans, 2nd edition. $J$ Phys Act Health 2018:1-11.

47 Cornelissen VA, Fagard RH, Coeckelberghs E, et al. Impact of resistance training on blood pressure and other cardiovascular risk factors: a meta-analysis of randomized, controlled trials. Hypertension 2011;58:950-8.

48 Xiong X, Wang P, Li S, et al. Effect of Baduanjin exercise for hypertension: a systematic review and meta-analysis of randomized controlled trials. Maturitas 2015;80:370-8.

49 Casonatto J, Goessler KF, Cornelissen VA, et al. The blood pressurelowering effect of a single bout of resistance exercise: a systematic review and meta-analysis of randomised controlled trials. Eur J Prev Cardiol 2016;23:1700-14.

$50 \mathrm{Jin} \mathrm{X}, \mathrm{Pan} \mathrm{B}, \mathrm{Wu} \mathrm{H}$, et al. The effects of traditional Chinese exercise on hypertension: a systematic review and meta-analysis of randomized controlled trials. Medicine 2019;98:e14049.

51 MacDonald HV, Johnson BT, Huedo-Medina TB, et al. Dynamic resistance training as stand-alone antihypertensive lifestyle therapy: a meta-analysis. J Am Heart Assoc 2016;5. doi:10.1161/ JAHA.116.003231. [Epub ahead of print: 28 Sep 2016].

52 Corso LML, Macdonald HV, Johnson BT, et al. Is concurrent training efficacious antihypertensive therapy? A meta-analysis. Med Sci Sports Exerc 2016;48:2398-406.

53 Cornelissen VA, Smart NA. Exercise training for blood pressure: a systematic review and meta-analysis. J Am Heart Assoc 2013;2:e004473.

54 Igarashi Y, Akazawa N, Maeda S. Regular aerobic exercise and blood pressure in East Asians: a meta-analysis of randomized controlled trials. Clin Exp Hypertens 2018;40:378-89.

55 Wang J, Feng B, Yang X, et al. Tai chi for essential hypertension. Evid Based Complement Alternat Med 2013;2013:1-10.

56 Xiong X, Wang P, Li X, et al. Qigong for hypertension: a systematic review. Medicine 2015;94:e352.

$57 \mathrm{Wu}$ Y, Johnson BT, Acabchuk RL, et al. Yoga as antihypertensive lifestyle therapy: a systematic review and meta-analysis. Mayo Clin Proc 2019;94:432-46.

58 Zhong D, Li J, Yang H, et al. Tai chi for essential hypertension: a systematic review of randomized controlled trials. Curr Hypertens Rep 2020;22:25-020-1031. 
59 Wu Y, Johnson BT, Chen S, et al. Tai Ji Quan as antihypertensive lifestyle therapy: a systematic review and meta-analysis. $J$ Sport Health Sci 2020:1:S2095-2546(20)30038-7.

60 Sheppard JP. Should exercise be considered as an alternative to drug treatment in patients with low-risk mild hypertension? $\mathrm{Br} J$ Sports Med 2019;53:848-9.

61 Brown RE, Riddell MC, Macpherson AK, et al. The joint association of physical activity, blood-pressure control, and pharmacologic treatment of hypertension for all-cause mortality risk. Am J Hypertens 2013;26:1005-10.

62 Lavie CJ, Stewart M, Ozemek C. Benefits of exercise training on blood pressure and beyond in cardiovascular diseases. Eur J Prev Cardiol 2020;27:244-6.

63 Pescatello LS. Exercise measures up to medication as antihypertensive therapy: its value has long been underestimated. $\mathrm{Br}$ J Sports Med 2019;53:849-852.
64 Unger T, Borghi C, Charchar F, et al. 2020 International Society of hypertension global hypertension practice guidelines. Hypertension 2020;75:1334-57.

65 Williams B, Mancia G, Spiering W, et al. 2018 ESC/ESH guidelines for the management of arterial hypertension. Eur Heart $J$ 2018;39:3021-104.

66 U.S. Department of Health and Human Services. The surgeon general's call to action to control hypertension. 48. Washington, D.C.: U.S. Department of Health and Human Services, Office of the Surgeon General, 2020.

67 Wu Y, Pescatello LS. The clinical utility of neuromotor exercise as antihypertensive lifestyle therapy. Curr Sports Med Rep 2020;19:133-6.

68 Pescatello LS. What's new in the ACSM pronouncement on exercise and hypertension? 2019. Available: https://www.acsm.org/home/ featured-blogs-homepage/acsm-blog/2019/06/11/new-acsmpronouncement-exercise-hypertension 